\title{
Next Generation Sequencing Reveals
}

a Synchronous Trilateral Lung Adenocarcinoma

Case with Distinct Driver Alterations of EGFR 19

Deletion or EGFR 20 Insertion or EZR-ROSI

Fusion

This article was published in the following Dove Press journal:

OncoTargets and Therapy

Xuhui Zhang ${ }^{1, *}$

Jiemei Feng ${ }^{2, *}$

Xiaoxing $\mathrm{Su}^{3}$

Yan Lei ${ }^{3}$

Wendy Wu (1) $^{3}$

Xiangyang Cheng ${ }^{4}$

'Department of Oncology, Guangdong Second Provincial General Hospital, Guangzhou, People's Republic of China; ${ }^{2}$ Department of Respiratory and ICU, Guigang People's Hospital, Guigang, 537100, People's Republic of China; ${ }^{3}$ Clinical Research Division, Berry Oncology Corporation, Fuzhou, 350200, People's Republic of China; ${ }^{4}$ Department of Thoracic Surgery, The First Affiliated Hospital of Guangzhou Medical University, Guangzhou, 510120, People's Republic of China

*These authors contributed equally to this work
Correspondence: Wendy Wu;

Xiangyang Cheng

Email wujy3261@berryoncology.com;

xuewul90I@sina.com
Objective: Synchronous multiple primary lung cancer (SMPLC) has a reported occurrence from $0.5 \%$ to $2 \%$ in lung cancer, and the surgical treatment and prognosis were quite diverse. With the discovery of driver mutations in lung adenocarcinoma (ADC), next-generation sequencing (NGS) would provide an explicit answer to the key question, whether individual tumors represent intrapulmonary metastases or independent tumors. Here, we reported a 64year-old female diagnosed with a synchronous trilateral early-stage ADC with distinct driver alterations.

Materials and Methods: NGS test targeting 31 cancer-relevant genes and amplification RNA sequencing (if gene fusion was found on DNA level) were performed on the surgical tumor tissue.

Results: A 64-year-old Chinese female never smoker was found with one nodule in the right upper lobe and two nodules in the right middle lobe through chest computed tomography. The lesions were resected through video-assisted thoracic surgery and diagnosed with stage IA ADC, T1N0M0, in the postoperative pathology. NGS detected three independent driver mutations in three primary sites, respectively, EGFR 19del, EGFR 20ins and ROS1 fusion. Conclusion: This is the first report of a synchronous trilateral early-stage ADC with distinct driver alterations. All individual tumors were independent identified by NGS methodology, which had provided a clear answer to the key question of SMPLC in this case and should be used as a routine genetic test to explore fully pathological diagnosis and more comprehensive oncogenesis information in the early-stage ADC clinical prevention.

Keywords: synchronous multiple primary lung cancer, lung adenocarcinoma, NGS, EGFR mutation, ROS1 fusion

\section{Introduction}

The incidence of adenocarcinoma (ADC) accounts for nearly $50 \%$ of all lung cancer, which remains the leading cause of cancer death (1.8 million, 18.4\%) and occupies the highest number of new cancer cases (2.1 million, $11.6 \%$ ) worldwide. ${ }^{1}$ Synchronous multiple primary lung cancer (SMPLC) has a reported occurrence from $0.5 \%$ to $2 \%$ in lung cancer patients, ${ }^{2}$ which has increased recently due to the development of early detection techniques. Based on the established clinicopathological criteria, the surgical 
outcome and prognosis of SMPLC patients were quite different even if all the lesions were ADC. ${ }^{3}$ With the discovery of driver mutations in ADC, such as epidermal growth factor receptor $(E G F R)$ mutation, $A L K$ receptor tyrosine kinase $(A L K)$ and c-ros oncogene 1 (receptor tyrosine kinase, ROS1) rearrangement, ${ }^{4}$ next-generation sequencing (NGS) would provide a more clear answer to the key question, whether individual tumors represent intrapulmonary metastases or independent tumors. Liu et al had profiled genomic heterogeneity of 6 SMPLC patients and suggested that different lung cancer in the same patient may have distinct genomic profiles and could be driven by distinct molecular events. ${ }^{5}$ There were 2 pN0 trilateral cases in this study, one patient had EGFR p.L858R mutation in two nidi and $P I K 3 C A$ mutations in the third nidus, while another patient had EGFR p.L858R mutation in all lesions. Luo et al reported a double $A L K$ rearrangement bilateral lung adenocarcinoma case in recent, one nodule with $P R K C B-A L K$ fusion and the other one with EML4-ALK fusion. ${ }^{6}$

EGFR and ROS1 are both well-established driver alterations with $10 \sim 35 \%$ and $1 \%$ prevalence, respectively, in non-small cell lung cancer (NSCLC) patients, and preferentially affecting non-smokers., ${ }^{4,7}$ Compound EGFR mutations are defined as double or multiple mutations in tyrosine kinase domain of EGFR, which are frequently detected in advanced lung cancer. In a retrospective study of 3000 treatment-naïve Chinese advanced NSCLC patients, ${ }^{8}$ compound $E G F R$ mutations were found in $1.2 \%$ EGFR mutated patients; none EGFR exon 19 deletion (19del) cases was observed in the compound $E G F R$ dataset; ROS1 fusion had not been detected in all $1266 E G F R$ mutation patients ( $42.2 \%$ of the whole cohort). ROS1 rearrangement had been identified with several 5 ' fusion gene partners, such as Ezrin $(E Z R)$, and ROS1 fusion is mostly exclusive to $E G F R, K R A S$, or $A L K$ mutations. $^{7}$ The concurrent ROS1 rearrangement with EGFR mutation in a single tumor was only observed in 6 cases, all were advanced ADC patients, and one of them was EZR-ROS1 fusion with EGFR 19del. ${ }^{9-12}$

Here we described an extremely rare case that a 64 years old female never-smoker early-stage ADC patient had three synchronous primary adenocarcinoma nodules with distinct driver mutations of EGFR 19del (stage IA, T1N0M0) or EGFR exon 20 insertion (20ins, stage IA, T1N0M0) or EZR-ROS1 fusion (stage IA, T1N0M0). All individual tumors were independent. NGS had provided a clear differentiation in this case and offered an explicit answer to the key question of SMPLC, whether individual tumors represent intrapulmonary metastases or independent tumors. NGS technique should be used as a routine genetic test to explore fully pathological diagnosis and more comprehensive tumorigenesis information for ADC patients.

\section{Materials and Methods}

The patient underwent resection of the right middle lobe and wedge resection of the right upper lobe and en bloc resection of the associated hilar and mediastinal lymph nodes by video-assisted thoracic surgery. Genomic DNA (gDNA) was extracted from fresh surgical tumor tissue samples using DNeasy Blood \& Tissue Kit (Qiagen, USA) according to the manufacturer's instructions, and the paired white blood cell samples using DNA Blood Midi/ Mini kit (Qiagen, USA). An in-house designed panel targeting 31 cancer-relevant genes (Berry Oncology) was used to generate sequencing libraries, which was sequenced by NextSeq CN500 (Berry Genomics, China \& Illumina, USA) with 150PE mode. Amplification RNA sequencing (if gene fusion was found on DNA level) was performed on the surgical tumor tissue.

\section{Case Presentation}

A 64-year-old non-smoking female was diagnosed as multiple pulmonary nodules in lung through physical examination and visited our hospital for follow-up with no abnormality in June, 2019. Chest computed tomography (CT) revealed one lesion in the right upper lobe and two lesions in the right middle lobe on June 16, 2019 (Figure $1 \mathrm{~A}, \mathrm{C}$ and $\mathrm{E}$ ). The subsequent surgical resection had been performed through video-assisted thoracic surgery (VATS). The post-operative course was uneventful and the patient recovered quickly. Immunohistochemical staining in the post-operative pathology indicated that three nodules of the patient all was clinically diagnosed with stage IA minimally invasive lung adenocarcinoma, T1N0M0 (Figure 1B, D and F).

The surgical tumor tissues were subjected to target capture-based clinical next-generation sequencing (NGS) test targeting 31 cancer-relevant genes (Berry Oncology), and three independent driver mutations had been found in three nidi, respectively (Figure 2). EGFR exon 19 deletion (c.2240-2260delinsCAAGAG) (Figure 2A) was detected at a mutant allele frequency (MAF) of $1.13 \%$ in the nidus $(0.5 \mathrm{~cm}$ in diameter) of the right upper lobe (Figure 1A). EGFR exon 20 insertion (c.2311-2312delinsGGGTT) (Figure 2A) was identified at a MAF of $5.13 \%$ in one 
A

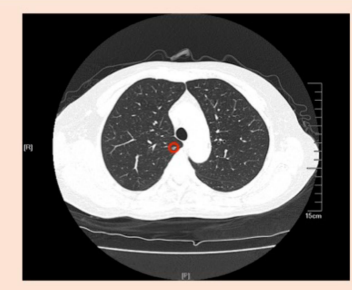

B

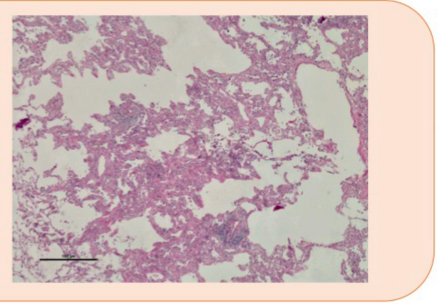

C

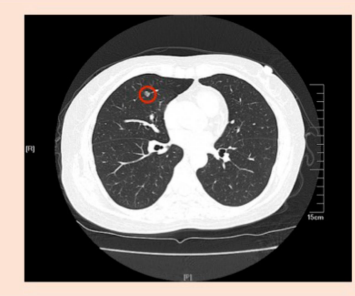

E

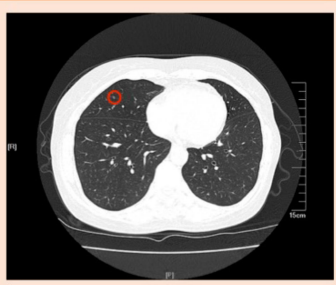

D

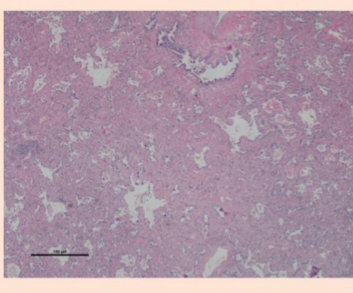

$\mathbf{F}$

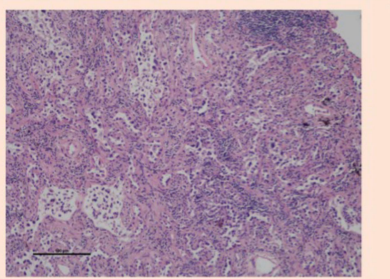

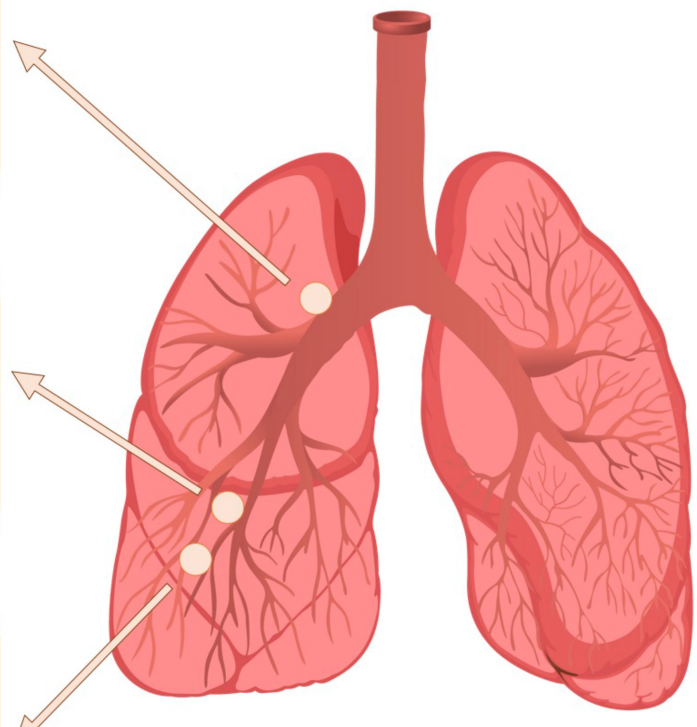

Figure I Chest computed tomography (CT) for the nodules in the lung lobes and the hematoxylin-eosin staining revealed the lung adenocarcinoma cells in the three primary sites $(100 \mu \mathrm{m})$. Three light red circles show the schematic location of the nodules. (A) and (B) for the nidus in the right upper lobe carrying EGFR I9del, (C) and (D) for the nidus in the right middle lobe carrying EGFR 20ins, and (E) and (F) for the nidus in the right middle lobe carrying ROSI fusion.

A

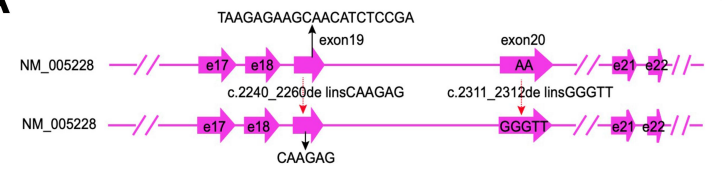

B

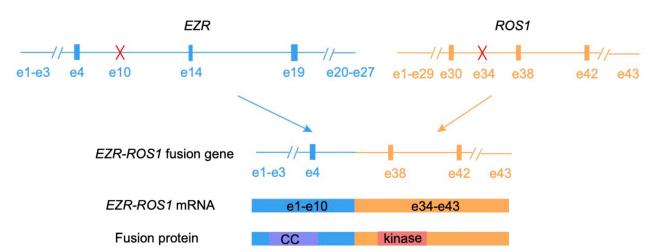

C
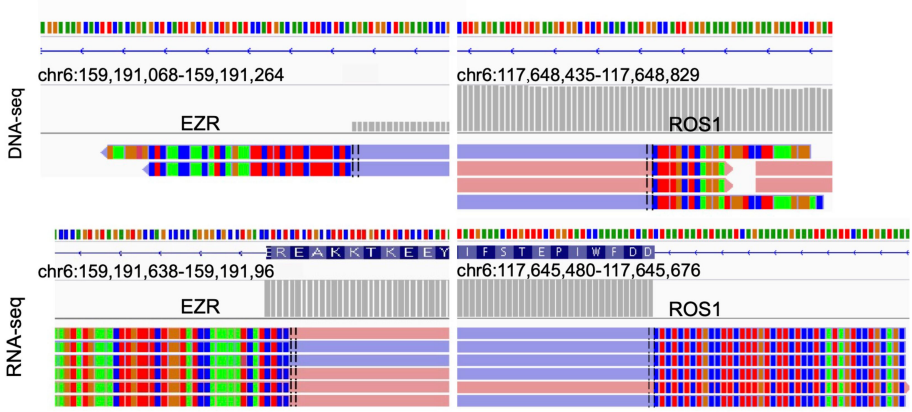

Figure 2 NGS targeting gene test on the trilateral surgical tumor tissue. (A) Schematic diagram of EGFR I9del and 20ins. (B) Schematic diagram of EZR-ROSI fusion. (C) Integrative Genomics Viewer (IGV) snapshot of EZR-ROSI fusion regions, which was detected by NGS sequencing reads.

nodule $(0.4 \mathrm{~cm}$ in diameter $)$ in the right middle lobe (Figure 1C). And EZR exon 10-ROS1 exon 34 (EZRROS1) rearrangement (Figure 2B) was detected at DNA level in the other nodule $(0.6 \mathrm{~cm}$ in diameter) in the right middle lobe (Figure 1E) and confirmed by amplification RNA sequencing with 7574 mutation fragments (Figure 2C). The fusion gene contained the $\mathrm{N}$-terminal coiled-coil domain of EZR and the C-terminal tyrosine kinase domain of ROS1. All sequencing reads were examined on Integrative Genomic Viewer (IGV) software.
Up to the 9 months post-surgery follow-up (until March 2020), no recurrence or metastasis was observed in the patient.

\section{Discussion}

EGFR gene is the most frequent driver mutation in $\mathrm{ADC},{ }^{4}$ and most alterations are located at the tyrosine kinase domain in exons 19 21. Short in-frame deletion in exon 19 and insertion in exon 20 are both typical mutations and have not been simultaneously found in two independent 
primary nidi of one pN0 lung adenocarcinoma patient in the literatures. ROS1 gene belongs to the insulin receptor family and the rearrangement with other gene partners would stimulate the downstream mitogen-activated protein kinases pathway, enhancing cell growth and proliferation. EZR-ROS1 fusion results in a not-so-small deletion of the long arm of chromosome $6,{ }^{7}$ which could keep the full ROS1 kinase domains and lead to constitutive kinase activity in tumor formation.

Previous investigations indicated that ROS1 rearrangement is hardly concurring with EGFR, KRAS, or $A L K$ mutations. Until now, ROS1 fusion was only observed to co-occur with EGFR alteration in 6 advanced ADC patients, ${ }^{9-12}$ all happened in a single tumor, one of them was the synchronous EZR-ROS1 fusion and EGFR 19del in the same lesion. Although compound EGFR mutations are frequently found in advanced ADC cases, one of the most prevalent EGFR mutations, EGFR 19del, is mostly exclusive. ${ }^{8}$ The concurrence of ROS1 rearrangement and $E G F R$ mutation has been detected in the same nidus of only one early-stage ADC patient, ${ }^{10}$ neither in the differential nidi. Here, we reported a scarce case carrying three primary tumors each with an independent driver mutation. It is the first time to discover this phenomenon in such an early-stage ADC case (stage IA, T1N0M0), which expanded our cognition of tumorigenesis.

This is the first report of a synchronous trilateral earlystage lung adenocarcinoma with distinct driver alterations of EGFR 19del or EGFR 20ins or EZR-ROS1 fusion. Three primary minimally invasive adenocarcinoma were surgical resected through VATS, no metastasis was found. All oncogenic mutations were identified by NGS methodology, DNA copy number variation (CNV) analysis was also performed but no amplification/deletion was found. Three nodules in this pN0 ADC patient have EGFR 19del, EGFR 20ins and EZR-ROS1 fusion, respectively, with sufficient genomic evidence. SMPLC only occurs $0.5 \%$ to $2 \%$ in lung cancer patients even with the worldwide use of high-resolution imaging systems, and the surgical treatment and prognosis of SMPLC are all related to accurate pathological diagnosis with molecular and genomics methods. In previous studies of synchronous primary lung adenocarcinoma, few cases with multiple tumors $(n \geq 3)$ were confirmed by NGS technology that they were independent tumors with distinct driver mutations. Our report identified that the patient had three different wellestablished genomic alterations in three separate nodules of two right lobes with NGS methodology. It could be concluded that three lesions with differential driver mutations were all independent tumors not intrapulmonary metastases. There is no abnormality presented on this patient from VATS surgery until now. EGFR tyrosine kinase inhibitors (EGFR-TKI) and crizotinib, the wellestablished Anaplastic Lymphoma Kinase $(A L K)$ inhibitor sensitive to ROS1 rearrangement, should be considered as the drug treatment when this case recurs.

Since $E G F R$ mutations are more frequent in lung adenocarcinoma with recurrent/metastatic disease, ${ }^{13}$ followup monitoring on this patient is needed with close attention. NGS technique had clearly differentiated three independent tumors in this SMPLC case and provided valuable insights into oncogenesis and early-stage cancer prevention in clinic. It suggested that differential malignant lung tumors in the same individual could have distinct genomic profiles and be driven by distinct molecular events under the identical genetic background. NGS detection should be used as a routine genetic test to explore fully molecular diagnosis and more comprehensive tumorigenesis information. We recommended that the genomic profiles of each resected nodule should be addressed through NGS method, which could expand the existing clinical practice solution for better outcome and precisely dynamics monitoring for $\mathrm{ADC}$ patients.

\section{Abbreviations}

SMPLC, synchronous multiple primary lung cancer; ADC, lung adenocarcinoma; NGS, next-generation sequencing; CT, computed tomography; VATS, video-assisted thoracic surgery; EGFR, epidermal growth factor receptor; 19del, exon 19 deletion; 20ins, exon 20 insertion; ROS1, c-ros oncogene 1; EZR, Ezrin.

\section{Ethics Statement}

The study was approved by the Ethical Committee of Medical Research, The First Affiliated Hospital of Guangzhou Medical University.

\section{Consent for Publication}

Written informed consent was obtained from the patient for publication of this case report and any accompanying images.

\section{Acknowledgments}

We thank Dr Lin Wu for her critical comments on the preparation of this manuscript. 


\section{Author Contributions}

All authors made a significant contribution to the work reported, whether that is in the conception, study design, execution, acquisition of data, analysis and interpretation, or in all these areas; took part in drafting, revising or critically reviewing the article; gave final approval of the version to be published; have agreed on the journal to which the article has been submitted; and agree to be accountable for all aspects of the work.

\section{Funding}

This work was partly supported by grants from National Natural Science Foundation of China (No.81973718).

\section{Disclosure}

Xiaoxing $\mathrm{Su}$, Yan Lei and Wendy $\mathrm{Wu}$ are employees of Berry Oncology Corporation. The authors report no other potential conflicts of interest for this work.

\section{References}

1. Bray F, Ferlay J, Soerjomataram I, Siegel RL, Torre LA, Jemal A. Global cancer statistics 2018: GLOBOCAN estimates of incidence and mortality worldwide for 36 cancers in 185 countries. CA Cancer J Clin. 2018;68(6):394-424. doi:10.3322/caac.21492

2. Synchronous Bilateral Lung Cancer With Discordant Histology. Available from: https://www.cancernetwork.com/lung-cancer/synchro nous-bilateral-lung-cancer-discordant-histology. Accessed November 27, 2020.
3. Loukeri AA, Kampolis CF, Ntokou A, Tsoukalas G, Syrigos K. Metachronous and synchronous primary lung cancers: diagnostic aspects, surgical treatment, and prognosis. Clin Lung Cancer. 2015;16(1):15-23. doi:10.1016/j.cllc.2014.07.001

4. Black RC, Khurshid H. NSCLC: an update of driver mutations, their role in pathogenesis and clinical significance. R I Med J. 2015;98 (10):25-28.

5. Liu Y, Zhang J, Li L, et al. Genomic heterogeneity of multiple synchronous lung cancer. Nat Commun. 2016;7:13200.

6. Luo J, Gu D, Lu H, Liu S, Kong J. Coexistence of a novel PRKCB-ALK, EML4-ALK double-fusion in a lung adenocarcinoma patient and response to crizotinib. J Thorac Oncol. 2019;14(12): e266-e268. doi:10.1016/j.jtho.2019.07.021

7. Takeuchi K, Soda M, Togashi Y, et al. RET, ROS1 and ALK fusions in lung cancer. Nat Med. 2012;18(3):378-381. doi:10.1038/nm.2658

8. Li M, Zhou CZ, Yang JJ, et al. The in cis compound EGFR mutations in Chinese advanced non-small cell lung cancer patients. Cancer Biol Ther. 2019;20(8):1097-1104.

9. Facchinetti F, Loriot Y, Kuo MS, et al. Crizotinib-resistant ROS1 mutations reveal a predictive kinase inhibitor sensitivity model for ROS1- and ALK-rearranged lung cancers. Clin Cancer Res. 2016;22 (24):5983-5991. doi:10.1158/1078-0432.CCR-16-0917

10. Zhu YC, Xu CW, Ye XQ, et al. Lung cancer with concurrent EGFR mutation and ROS1 rearrangement: a case report and review of the literature. Onco Targets Ther. 2016;9:4301-4305.

11. Ju L, Han M, Zhao C, Li X. EGFR, KRAS and ROS1 variants coexist in a lung adenocarcinoma patient. Lung Cancer. 2016;95:94-97. doi:10.1016/j.lungcan.2016.03.005

12. Mao Y, Wu S. ALK and ROS1 concurrent with EGFR mutation in patients with lung adenocarcinoma. Onco Targets Ther. 2017;10:3399-3404. doi:10.2147/OTT.S133349

13. Jordan EJ, Kim HR, Arcila ME, et al. Prospective comprehensive molecular characterization of lung adenocarcinomas for efficient patient matching to approved and emerging therapies. Cancer Discov. 2017;7(6):596-609. doi:10.1158/2159-8290.CD-16-1337
OncoTargets and Therapy

\section{Publish your work in this journal}

OncoTargets and Therapy is an international, peer-reviewed, open access journal focusing on the pathological basis of all cancers, potential targets for therapy and treatment protocols employed to improve the management of cancer patients. The journal also focuses on the impact of management programs and new therapeutic agents and protocols on patient perspectives such as quality of life, adherence and satisfaction. The manuscript management system is completely online and includes a very quick and fair peer-review system, which is all easy to use. Visit http://www.dovepress.com/ testimonials.php to read real quotes from published authors. 